\title{
Steady-state performance of microcavity surface-emitting lasers with quantum confinement of electrons and photons
}

Igor Vurgaftman and Jasprit Singh

Solid State Electronics Laböratory, Department of Electrical Engineering and Computer Sciences, The University of Michigan, Ann Arbor, Michigan 48109-2122

(Received 22 November 1993; accepted for publication 17 January 1994)

\begin{abstract}
In conventional surface-emitting lasers, the photon density of states is essentially that of a bulk material and, therefore, spontaneous emission rates are the same as in bulk. However, in an ideal laser, one would like to have a single photon mode coincident with the peak in the material gain so that zero-threshold lasing occurs. This possibility is examined for a surface-emitting microcavity laser. The photon density of states for realistic GaAs-based microcavity structures ( $4 \mu \mathrm{m}$ length and $0.5-4 \mu \mathrm{m}$ lateral width) is derived from solutions to the wave equation in the cavity. This density of states is used to study the properties of microcavity lasers with compressively strained quantum well active media by using the exact form of spontaneous emission rate in the ratc cquations. It is found that when the lateral dimension approaches $0.5 \mu \mathrm{m}$, all optical power is present in a single resonant photon mode (the lasing mode), although additional leaky and propagating modes exist. The spontaneous emission factors and threshold current densities are derived for a few microcavity lasers. It is found that for microcavities with lateral dimensions and aspect ratios practical in experimental realization, the ideal "thresholdless" behavior is not observed, although the threshold current density is reduced to $\approx 60 \mathrm{~A} / \mathrm{cm}^{2}$ compared with $160 \mathrm{~A} / \mathrm{cm}^{2}$ calculated for lasers with cross sections typical for conventional surface-emitting lasers.
\end{abstract}

In conventional semiconductor lasers, the photon density of states is essentially that of a bulk system, and, as a result, a large fraction of electron-hole pairs emit photons into nonlasing modes. This high spontaneous emission rate is undesirable from the point of view of laser performance. The spontaneous emission lifetime in an optical cavity can be modified by reducing the size of the cavity to the order of the photon wavelength. ${ }^{1}$ Changes in the spontaneous emission rate reflect the altered photon density of states which encompasses only allowed cavity modes. Ideally, maximum efficiency of the mechanism of spontaneous emission can be achieved with a single mode overlapping the material gain spectrum of the active medium. Thus, an ideal microcavity semiconductor laser exhibits complete absence of the threshold transition between incoherent, spontaneous-emissiondominated and coherent, stimulated-emission-dominated optical output, as well as significantly improved modulation characteristics. ${ }^{2}$ Several practical realizations of microcavity lasers have been proposed. In this letter, the subject of our investigation is a microcavity laser obtained by shrinking the lateral width of the surface-emitting laser in which the vertical cavity is formed by a pair of distributed Bragg reflectors (DBR) composed of quarter-wavelength stacks of materials with different refractive indices (see Fig. 1). The mirrors are separated by an integral number of half-wavelengths, and a standing-wave mode is formed owing to the phase slip in an otherwise periodic structure. This Bragg resonant mode can be most conveniently regarded as a bound state in the photonic forbidden gap localized to the quarter-wavelength defect in the periodic structure. ${ }^{3}$ In order to optimize the threshold-current performance of our microcavity laser, the active medium is assumed to be comprised of a single compressively strained quantum well. In this letter, we consider the steady-state performance characteristics of the microcav- ity lasers based on a rigorous analysis of confined electrons and photons.

The material gain spectrum in the active medium can be calculated using the Fermi Golden Rule and a knowledge of the conduction- and valence-band structure. ${ }^{4}$ A 4-band $\mathbf{k} \cdot \mathbf{p}$ method in conjunction with the deformation potential theory is used to describe the valence band states. Since light polarized perpendicular to the direction of epitaxial growth couples solely to electron-light hole transitions, the gain for this polarization is suppressed in the vicinity of the band edge. All calculations in this letter are done for $a_{1} 50 \AA$ $\mathrm{In}_{0.2} \mathrm{Ga}_{0.8}$ As quantum well.

In order to solve for the optical modes in the microcavity, an assumption is made that the wave equation is sepa-

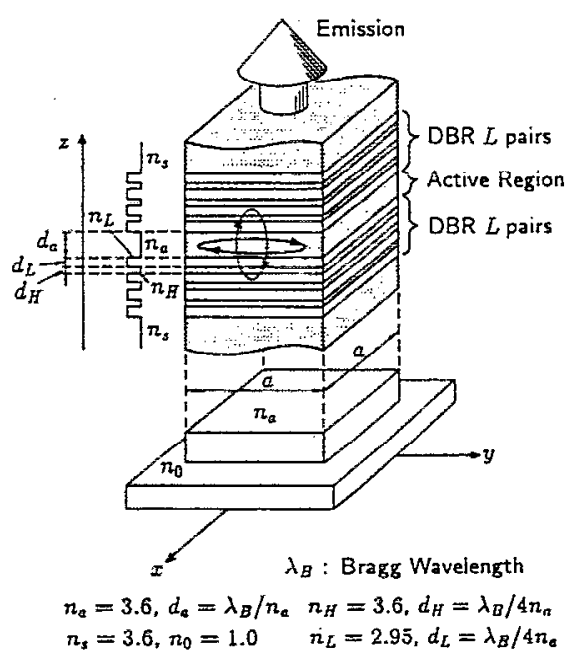

FIG. 1. 'The vertical-cavity surface-emitting microcavity structure. Reproduced from Ref. 5. 
rable in the vertical and horizontal coordinates. ${ }^{5}$ The photon dispersions in the vertical cavity are readily derived by the transfer matrix technique. ${ }^{5}$ Three classes of modes are recovered. Besides the Bragg resonant modes, there will be leaky modes located beyond the forbidden gap and essentially unconfined by the cavity. Also, for a large transverse component of the photon wave vector, it is possible to have modes propagating in the horizontal direction.

The modes in the horizontal cavity can be obtained analytically or via a numerical solution of the two-dimensional (2D) wave equation depending upon the cavity geometry. For a rectangular cross section, the solutions are sines and cosines, while for a circular cross section, they are given by Bessel functions of various orders. For simplicity, only square cross sections are considered further. Adding the longitudinal and transverse components of the incident angle, it is reasonable to assume that all modes in the horizontal cavity suffer total internal reflection and are discrete. Modes in the $3 \mathrm{D}$ cavity are given by intersections of the modes in the vertical and horizontal cavities. The microcavity considered in this letter consists of 15.5 pairs of GaAs/AlAs stacks with a wavelength-long central region and transverse dimensions in the range from $2 \lambda_{B} / n_{a}-16 \lambda_{B} / n_{a}$, where $\lambda_{B}$ is the Bragg wavelength and $n_{a}$, the refractive index of the active medium, or $\approx 0.5-4 \mu \mathrm{m}$ for a GaAs-based system.

Next the photon density of states is computed. The leaky modes are unconfined by the cavity and contribute a 1D density of states for each transverse solution. The line shape function for the resonant and propagating modes is assumed to be a Lorentzian with the linewidth $\Gamma=(\hbar c / n)\left[(1 / L) \ln (1 / R)+\alpha_{i}\right]$. The reflectivity $R$ is $\approx 32 \%$ for the modes propagating in the transverse direction. $R$ for the resonant modes must be calculated using a transfermatrix approach similar to that described above. ${ }^{6}$ Peaks in the reflectivity occur in the forbidden gap, in which the Bloch wave number acquires an imaginary component, and the Bloch wave becomes evanescent, and approximately coincides with the peaks in the density of states. Analytical results are available for normally incident radiation $(R$ $\approx 99.1 \%$ for this structure). The reflectivity increases with the transverse wavenumber for TE modes and decreases initially for TM modes, passing through zero at the Brewster angle. Note that it is particularly important to account correctly for the transverse variation in the reflectivity in a microcavity, where light may be incident at the surface at a large angle.

The photon density of states in a $0.5 \mu \mathrm{m}$ cavity is shown in Fig. 2. The formation of a staircase-like structure reminiscent of the electronic subbands in quantum wells can be observed. Our primary interest is in the vicinity of the first resonant peak. Note that despite the enhanced density of states at the peak, the overall density of states integrated over the region near the resonant peak is smaller than in the absence of the cavity. This is due to insufficient filling of the photon wave vector space by the discrete transverse modes. Although the first TM $\left(\mathrm{TM}_{00}\right)$ resonant mode is located close to the first $\mathrm{TE}\left(\mathrm{TE}_{00}\right)$ mode, only the latter is capable of lasing. The reason is twofold: (1) due to a lower reflectivity, $\mathrm{TM}_{00}$ is subject to a higher loss; (2)TM $\mathrm{TM}_{00}$ possesses a large

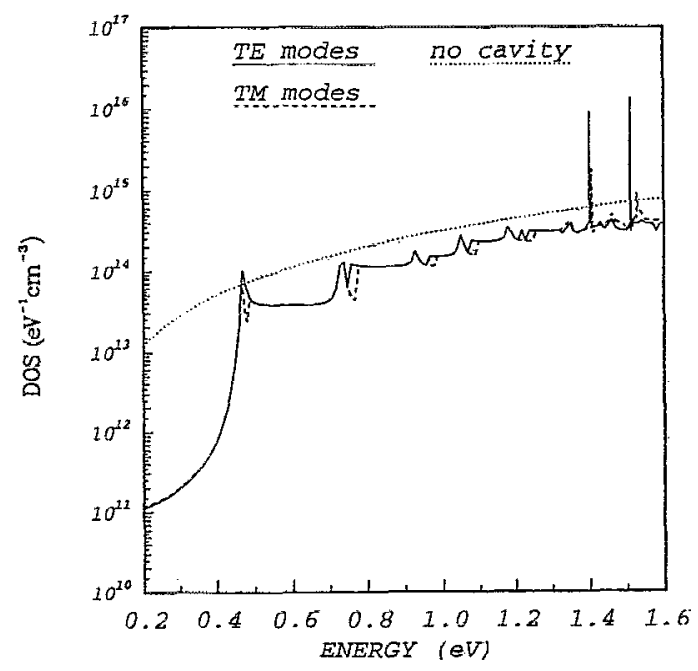

FIG. 2. The photon density of states for the microcavity structure in Fig. 1 for TE and TM polarized modes. The density of states in absence of a cavity is shown for comparison.

polarization component in the direction perpendicular to that of epitaxial growth, and material gain for that polarization is suppressed near the band edge. The microcavity laser is optimally designed by matching the peak in the material gain at threshold with $\mathrm{TE}_{00}$.

The spontaneous emission rate due to electron-hole pair recombination in a quantum-confined active region is given by (in Gaussian units):

$$
\begin{aligned}
R_{s p}= & \int d(\hbar \omega) \frac{4 \pi^{2} e^{2} \hbar}{n^{2} m_{0}^{2} \hbar \omega} \frac{1}{2 \pi^{2}} \sum_{n, m} \int d k \sum_{\sigma, \hat{\varepsilon}}\left|\hat{\varepsilon} \mathbf{P}_{n m}^{\sigma}(k)\right|^{2} \\
& \times \rho_{\varepsilon}(\hbar \omega) \delta\left(E_{n}^{e}(k)-E_{m}^{h}(k)-\hbar \omega\right) \\
& \times\left[f^{e}\left(E_{n}^{e}(k)\right)\right]\left[1-f^{h}\left(E_{m}^{h}(k)\right)\right]
\end{aligned}
$$

where $\rho_{\hat{\varepsilon}}$ is the (in general) polarization-dependent photon density of states. For a very large cavity, the photon density of states tends to the limit of the polarization-independent density of states:

$$
\rho(\hbar \omega)=\frac{2}{3} \frac{(\hbar \omega)^{2} n^{3}}{2 \pi^{2} c^{3} \hbar^{3}},
$$

where an averaging over the two degenerate polarizations has been performed. In our calculations, the previously derived density of states is used in Eq. (1). The spontaneous emission rate for the quantum well active region for TE and TM polarizations is shown in Fig. 3 and compared with the spontaneous emission rate in absence of the cavity. It is obvious that while spontaneous emission is enhanced around the resonant peak, it is suppressed elsewhere. As a result of this suppression, the spontaneous emission lifetime in the cavity increases.

The steady-state solutions of the multimode rate equations for the current density and the photon density can be studied to simulate the steady-state performance of microcavity lasers: 


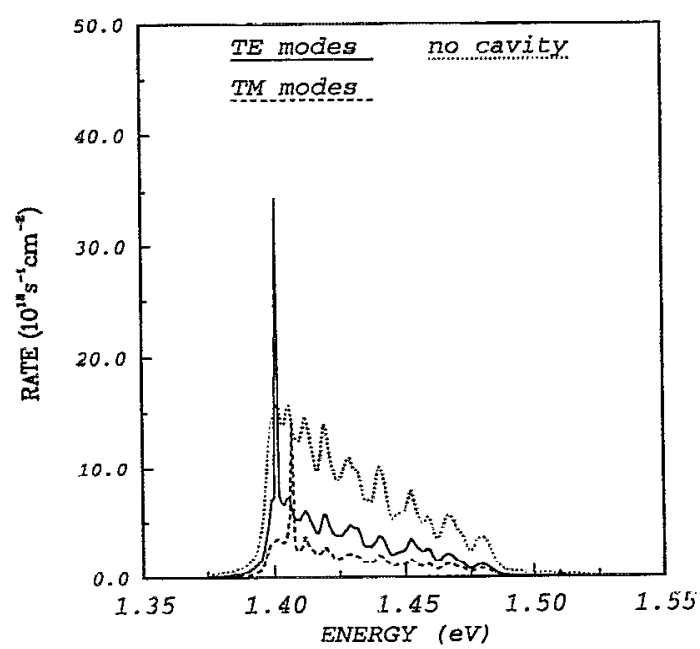

FIG. 3. The spontaneous emission rate at an injected current level of 200 $\mathrm{A} / \mathrm{cm}^{2}$. The spontancous cmission rate in absence of a cavity is shown for comparison.

$$
\begin{aligned}
& S_{\hat{\varepsilon}, m}=\frac{R_{s p, \hat{\varepsilon}, m}(N)}{\left(c / n_{g}\right)\left[\alpha_{c}-\Gamma g\left(N, E_{m}\right)\right]}, \\
& \frac{J}{e}=R_{s p}(N)\left(1+\sum_{m, \hat{\varepsilon}} \frac{R_{s p, \hat{\varepsilon} m}(N) \Gamma g\left(N, E_{m}\right)}{R_{s p}(N)\left[\alpha_{c}-\Gamma g\left(N, E_{m}\right)\right]}\right),
\end{aligned}
$$

where $S_{\hat{\varepsilon}, m}$ is the photon density per unit area in mode $m$ of $\hat{\varepsilon}$ polarization, $R_{s p, \hat{\varepsilon}, m}$ is the spontaneous emission rate into the mode, $R_{s p}=\Sigma_{\hat{\varepsilon}, m} R_{s p, \hat{\varepsilon}, m}$ is the total spontaneous emission rate, $\alpha_{c}$ is the total cavity loss, and $\Gamma$ is the optical confinement factor assumed to be proportional to the quantum well width for narrow wells, and taken to be $1.25 \%$ in these calculations. Note that in our formulation of the problem, there is no need to assume a certain value of the spontaneous emission coupling factor. This factor is found implicitly $\left(R_{s p, \hat{\varepsilon}, m} / R_{s p}\right)$ in the process of calculating the photon density of states and the recombination rate. We find that the factor is in principle different for distinct resonant modes. For a $2 \lambda_{B} / n_{a}$ cavity, it is $\approx 0.1$, decreasing to 0.05 for a $4 \lambda_{b} / n_{a}$ cavity. It is also important to point out that only various resonant modes need be included in the solution of the multimode rate equations. Any photons emitted into the leaky modes and propagating modes will immediately escape from the cavity.

The density of the photons in the cavity as a function of the injected current density is shown in Fig. 4 for several lateral cavity widths. As the lateral width is decreased to 0.5 $\mu \mathrm{m}$, nearly all photons present in the cavity have been emitted into the lasing mode, while for the $1 \mu \mathrm{m}$ cavity, this holds only for $60 \%-70 \%$ of all photons. The fraction of photons in the lasing mode declines with increasing cavity width reaching the limit of the conventional surface-emitting laser at $\approx 4 \mu \mathrm{m}$. Nevertheless, the curvature in the lightcurrent characteristic is well defined, and the threshold transition exists even for the $0.5 \mu \mathrm{m}$ cavity. The threshold current density is defined as the intersection of the extrapolated light-current curve in the linear regime with zero photon den-

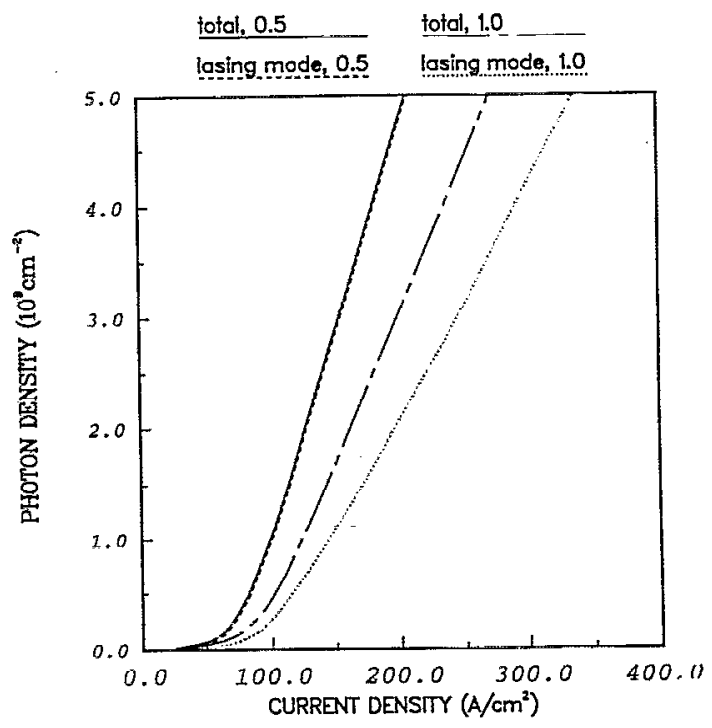

FIG. 4 . The photon density vs the injected current density for the $0.5 \mu \mathrm{m}$ and $1 \mu \mathrm{m}$ wide cavities in all modes and in the lasing mode only.

sity. The threshold current density is $\approx 60 \mathrm{~A} / \mathrm{cm}^{2}$ in the 0.5 $\mu \mathrm{m}$ cavity, $80 \mathrm{~A} / \mathrm{cm}^{2}$ in the $1.0 \mu \mathrm{m}$ cavity, $120 \mathrm{~A} / \mathrm{cm}^{2}$ in the $2.0 \mu \mathrm{m}$ cavity and $150 \mathrm{~A} / \mathrm{cm}^{2}$ in the $4.0 \mu \mathrm{m}$ cavity. With further increases in the cavity width, the threshold current density salurales at $\approx 160 \mathrm{~A} / \mathrm{cm}^{2}$ corresponding to the limit of the conventional surface-emitting laser.

In conclusion, we have considered the steady-state performance characteristics of microcavity lasers based on a rigorous coupled analysis of confined electrons and photons. Exact forms of the photon and electron dispersions and densities of states have been obtained from a numerical analysis of the electronic bandstructure, the wave equation in the cavity and reflectivities of the distributed mirrors. We have derived the spontaneous emission coupling factors and threshold current densities for a number of microcavity structures. The limit in which all photons in the cavity belong to the lasing mode thus substantially improving the large-signal modulation characteristics of microcavity lasers has been identified. In addition, it has been shown that for microcavity structures currently capable of experimental realization, the ideal "thresholdless" behavior is not exhibited owing to an insufficiently large value of the spontaneous emission coupling factor. Detailed studies of the steady-state and modulation characteristics of microcavity lasers will be presented in a more comprehensive article.

This work was supported by the Army Research Office under Contract No. DAAL 03-92-G-0109.

${ }^{1}$ F. DeMartini, G. Innocenti, G. R. Jacobovitz, and P. Mataloni, Phys. Rev. Lett. 59, 2955 (1987); E. Yablonovitch, T. J. Gmitter, and R. Bhatt, Phys. Rev. Lett. 61, 2546 (1988).

${ }^{2}$ Y. Yamamoto, S. Machida, and G. Bjork, Opt. Quantum. Electron. 24, S215 (1992); H. Yokoyama and S. D. Brorson, J. Appl. Phys. 66, 4801 (1989).

${ }^{3}$ E. Yablonovitch, Phys. Rev. Lett. 58, 2059 (1987).

${ }^{4}$ J. P. Loehr and J. Singh, IEEE J. Quantum. Electron. QE-27, 708 (1991).

${ }^{5}$ T. Baba, T. Hamano, F. Koyama, and K. Iga, IEEE J. Quantum Electron. QE-27, 1347 (1991); ibid. QE-28, 1310 (1992).

'See, for example, A. Yariv and P. Yeh, Optical Waves in Crystals (Wiley, New York, 1984), Chap. 6. 